\title{
Granuloma Piogênico em Lábio de Paciente Senil: Relato de Caso
}

Pyogenic Granuloma in the Lip of a Senile Patient: Case Report Granuloma Piógeno en Labio de Paciente Senil: Reporte de Caso

Gabrielly Melo BARBOSA

Acadêmica do curso de graduação em Odontologia, Escola Superior de Ciências da Saúde, Universidade do Estado do AmazonasUEA, 69065-001 Manaus-AM, Brasil Lioney Nobre CABRAL

Professor da Escola Superior de Ciências da Saúde, Universidade do Estado do Amazonas-UEA, 69065-001 Manaus-AM, Brasil, Doutor em Biotecnologia pela Universidade Federal do Amazonas-UFAM https://orcid.org/0000-0002-0505-4070 Antônio Jorge Araújo de VASCONCELOS II Professor da Escola Superior de Ciências da Saúde, Universidade do Estado do Amazonas-UEA, 69065-001 Manaus-AM, Brasil, Mestre em Patologia Bucal pela Universidade do Federal do Amazonas-UFAM https://orcid.org/0000-0001-5947-1438

\section{Resumo}

O granuloma piogênico é um crescimento de tecido conjuntivo que se assemelha a um tumor reativo, porém de natureza não neoplásica. A gengiva é a região mais acometida; entretanto, lábios, língua, e outras áreas da mucosa bucal e pele podem ser afetados, o trauma é predominante nesses locais. São encontrados com maior frequência na segunda e terceira década de vida, demonstrando predileção pelo gênero feminino. O presente trabalho relata o caso de granuloma piogênico em uma paciente de 62 anos. Durante a anamnese, a paciente relatou que o dente posterior traumatizava a região de lábio inferior, revelando o hábito parafuncional de mordiscamento do lábio, com tempo de evolução de, aproximadamente, 2 meses. Ao exame intraoral constatou-se a presença de lesão nodular exofítica, com base séssil, de coloração da mesma cor de mucosa labial circunjacente e levemente ulcerada. Diante dos dados clínicos, a hipótese diagnóstica foi de Mucocele. O tratamento realizado consistiu na remoção cirúrgica e, o espécime, encaminhado para o laboratório de patologia bucal da UEA, sendo o diagnóstico granuloma piogênico. A paciente foi acompanhada durante 2 anos e 3 meses no pós-operatório e não apresentou evidências de recidiva da lesão. À vista disso, este relato mostra a importância de conhecer as características das lesões mesmo quando a localização e idade do paciente são incomuns. Orientar e acompanhar o paciente, nesses casos, propicia um bom tratamento e prognóstico.

Descritores: Granuloma Piogênico; Lábio; Envelhecimento.

\section{Abstract}

Pyogenic granuloma is a growth of connective tissue that resembles a reactive tumor, but of a non-neoplastic nature. The gum is the most affected region; however, lips, tongue and other areas of the mouth mucosa and skin may be affected, trauma is prevalent in these places. They are found most frequently in the second and third decades of life, showing predilection for the female gender. The present study reports the case of pyogenic granuloma in a 62-year-old patient. During the anamnesis, the patient reported that the posterior tooth traumatized the lower lip region, revealing the parafunctional habit of lip biting, with evolution time of approximately 2 months. The intraoral examination showed the presence of exophytic nodular lesion, on a septic basis, of coloration of the same color as surrounding lip mucosa and lightly ulcerated. Before clinical data, the diagnostic hypothesis was Mucocele. The treatment carried out consisted in the surgical removal and, the specimen, forwarded to the laboratory of oral pathology of the UEA, being the diagnosis of pyogenic granuloma. The patient was accompanied for 2 years and 3 months in the postoperative and presented no evidence of recurrence of the lesion. In view of this, this account shows the importance of knowing the characteristics of the lesions even when the location and age of the patient are unusual. Guiding and accompanying the patient in such cases provides good treatment and prognosis.

Descriptors: Granuloma, Pyogenic; Lip; Aging.

\section{Resumen}

El granuloma es el crecimiento de un tejido conjuntivo que se asemeja a un tumor reactivo, sin embargo de naturaleza neoplásica. La encía es la región más afectada; entretanto labios, lengua y otras áreas de la mucosa bucal y piel pueden ser afectadas. El trauma es predominante en estas locaciones. Son encontrados con mayor frecuencia en la segunda y tercera década de vida, demonstrando preferencia por el género feminino. El trabajo actual relata el caso de granuloma piógeno en una paciente de 62 años. Durante la anamnesis, la paciente relató que el diente posterior traumatzaba la región del labio inferior, revelando el hábito parafuncional de mordisqueo del labio, con tiempo de evolución de, aproximadamente, 2 meses. En el examen intraoral se constató la presencia de una lesión nodular exofítica, con base sésil de la misma coloración de la mucosa labial circunyacente y levemente ulcerada. Frente a los datos clínicos, la hipótesis diagnosticada fue de Mucocele. El tratamento realizad consistió en la remoción quirúrgica y, el espécimen, fue encaminado al laboratorio de patologia bucal de la UEA, siendo el diagnóstico Granuloma piógeno. A la paciente se le hizo acompañamiento durante 2 años y 3 meses en revisión postquirúrgica y no presentó evidencia de recidiva de la lesión. En vista de esto, este relato muestra la importância de conocer las características de las lesiones aún cuando la localización y la edad del paciente son poco comunes. Orientar y acompañar al paciente, en estos casos, propicia un buen tratamiento y pronóstico.

Descriptores: Granuloma Piogénico; Labio; Envejecimiento.

INTRODUÇÃO

$$
\text { O granuloma piogênico (GP) é }
$$

considerado uma lesão benigna e comum da cavidade oral, sendo responsável por $3,81 \%$ a $7 \%$ de todas as biópsias ${ }^{1,2}$. O primeiro caso foi relatado por Hullihen em $1844^{3}$ e denominado como "granuloma piogênico" por Hartzell em $1904^{4}$. Inicialmente pensou-se que sua origem estivesse relacionada a organismos piogênicos, porém se constata agora não ser de origem infecciosa ${ }^{5}$ e que o termo granuloma piogênico é inadequado, visto que não há formação de exsudato purulento ${ }^{1,6}$.

Diversas etiologias têm sido consideradas para a formação do GP, como por exemplo, trauma de baixa intensidade, irritação crônica, alterações hormonais, medicamentos e má higiene bucal ${ }^{5}$. A mucosa mordiscada é uma condição causada por irritação física crônica, como mordida contínua da língua, lábios e 
mucosa ${ }^{8}$. Este frequente hábito parafuncional de mordiscar os tecidos bucais geram lesões que são mais comumente encontradas na mucosa jugal; contudo, a borda lateral da língua e a mucosa labial também são sítios afetados ${ }^{9}$.

Clinicamente, apresenta-se como uma lesão exofítica, eritematosa, séssil ou pediculada, com superfície lisa ou lobulada, indolor, de crescimento lento, frequentemente possui superfície ulcerada e tendência à sangramento espontâneo ao toque ${ }^{10}$. Os casos mais prevalentes são de localização gengival, todavia podem acometer os lábios, língua, mucosa jugal e outras áreas da mucosa bucal e pele ${ }^{11}$.

Essas lesões aparecem com mais frequência na maxila do que na mandíbula, sendo as áreas anteriores mais acometidas do que as áreas posteriores ${ }^{5}$. Esta condição pode afetar pessoas de qualquer faixa etária; entretanto, são mais encontradas na segunda e terceira década de vida, como nos adolescentes e adultos jovens; e mais, prevalentemente, em mulheres, possivelmente devido aos efeitos vasculares dos hormônios femininos estrogênio e progesterona que ocorrem durante a puberdade, gravidez e no uso de contraceptivos orais $^{12,13,14}$.

Devido ao aumento dos níveis desses hormônios (estrogênio e progesterona), ocorre uma dilatação e proliferação da microvasculatura gengival e destruição dos mastócitos, o que resulta em uma alta liberação de substâncias vasoativas que induzem a formação do granuloma ${ }^{15}$. A gravidez pode modular o metabolismo da progesterona e efetuar uma realocação das células inflamatórias no tecido, podendo contribuir para o estabelecimento de uma gengivite e posteriormente um granuloma, que neste estado recebe o nome de "granuloma gravídico"16.

Histologicamente, apresenta proliferação de tecido de granulação com infiltrado inflamatório misto de neutrófilos, plasmócitos e, especialmente, linfócitos e grande capacidade angiogênica ${ }^{7}$. Vários canais são formados e congestionados por hemácias, às vezes, organizando-se em agregados lobulares, a partir disso alguns patologistas usam dessa conformação para dar o diagnóstico de $\mathrm{GP}^{5}$. $\mathrm{O}$ diagnóstico diferencial inclui fibroma odontogênico periférico, hemangioma, angiossarcoma, angiomatose, granuloma de células gigantes periférico, fibroma ossificante periférico, linfoma não-Hodgkin e sarcoma de Kaposi ${ }^{7}$.

O tratamento da lesão, quando em gengiva, consiste em raspagem, alisamento radicular e biópsia por excisão que é a linha de tratamento recomendada. A excisão cirúrgica completa, usualmente, é curativa. Porém se for incompleta, se houver falha na remoção dos fatores etiológicos ou se ocorrer nova agressão da área a lesão pode recidivar ${ }^{5,17,18}$ e, conforme relatado por Bhaskar e Jacoway ${ }^{19}(1966)$, a taxa de recorrência é de $15,8 \%$. Outras formas de tratamento são os lasers cirúrgicos (de alta intensidade) $\mathrm{Nd}$ : YAG; $\mathrm{CO}_{2}{ }^{20}$ e laser pulsado ${ }^{21}$; a criocirurgia ${ }^{22}$ e escleroterapia com tetradecil sulfato de sódio $(\mathrm{STS})^{23}$.

A cavidade oral sofre constantemente estímulos internos e externos que, por vezes, são traumáticos, portanto, 0 diagnóstico e tratamento correto da lesão são significativos para a reabilitação. A localização da lesão e idade da paciente diferem do comumente relatado, isso justificando sua importância para a comunidade científica. Deste modo este trabalho visa relatar um caso clínico incomum de granuloma piogênico labial em uma paciente senil.

CASO CLÍNICO

Paciente do sexo feminino, 62 anos de idade, melanoderma, compareceu a policlínica odontológica da Universidade do Estado do Amazonas (UEA), à clínica de Estomatologia do curso de Odontologia, apresentando como queixa principal uma "bolinha no lábio", que, durante a alimentação, a incomodava. $\mathrm{Na}$ anamnese, a paciente relatou ser diabética, hipertensa e que o dente posterior (primeiro prémolar) traumatizava a região de lábio inferior do lado esquerdo, revelando o hábito, parafuncional, de mordiscamento do lábio, tendo evolução de, aproximadamente, 2 meses. Durante o exame clínico, constatou-se uma lesão nodular exofítica, com base séssil, de coloração semelhante à mucosa labial circunjacente, com superfície lisa, consistência firme e levemente ulcerada (Figura 1).

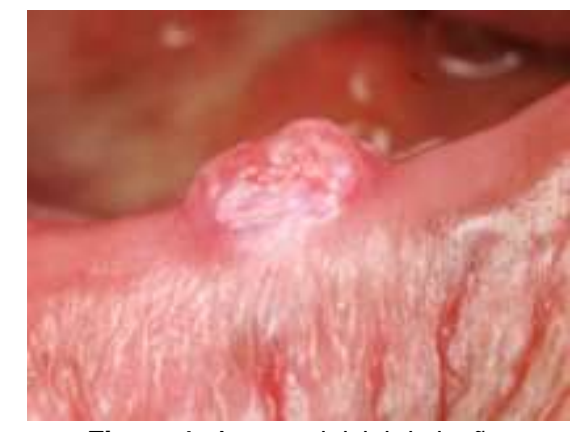

Figura 1: Aspecto inicial da lesão.

O tratamento de escolha foi a remoção cirúrgica. Para a cirurgia, foram solicitados previamente exames laboratoriais: hemograma, coagulograma e glicemia em jejum, que apresentaram valores normais. 
Realizou-se anestesia infiltrativa com lidocaína $2 \%$ à distância para não mascarar o volume da lesão. Com a lâmina 15, fez-se uma incisão de forma elíptica, após a incisão o tecido foi divulsionado, sempre margeando a lesão para verificar se havia atingido tecidos mais profundos, após a confirmação, a lesão foi totalmente excisionada medindo $1,0 \times 0,5 \times 0,2$ $\mathrm{cm}$ em suas maiores dimensões (Figura 2 ).

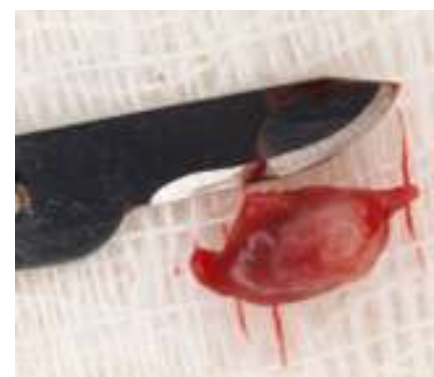

Figura 2: Espécime coletado para exame histopatológico.

Após a excisão da lesão a área cirúrgica foi suturada com fio 0.4 de nylon monofilamentado, sendo realizado três pontos simples (Figura 3).

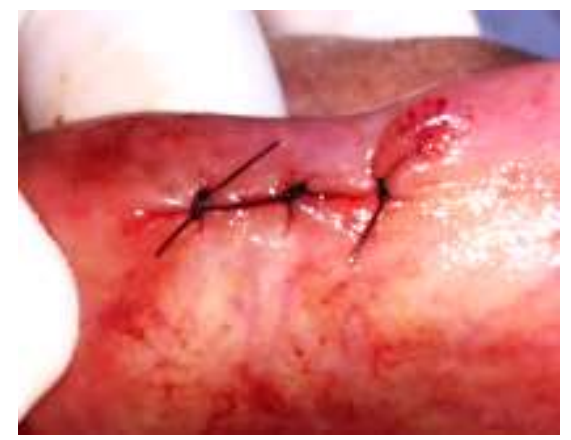

Figura 3: Síntese após biopsia excisional.

A lesão removida foi posta em formol a $10 \%$ e encaminhada para o laboratório de patologia bucal da UEA. Os cortes microscópicos revelaram fragmento de mucosa bucal revestida por epitélio estratificado pavimentoso paraqueratinizado hiperplásico (Figura 4 A), com intensa exocitose de leucócitos polimorfonucleares (Figura 4 B) e subjacente, tecido conjuntivo frouxo não modelado, rico em capilares congestos (Figura 5). Sendo o diagnóstico granuloma piogênico.

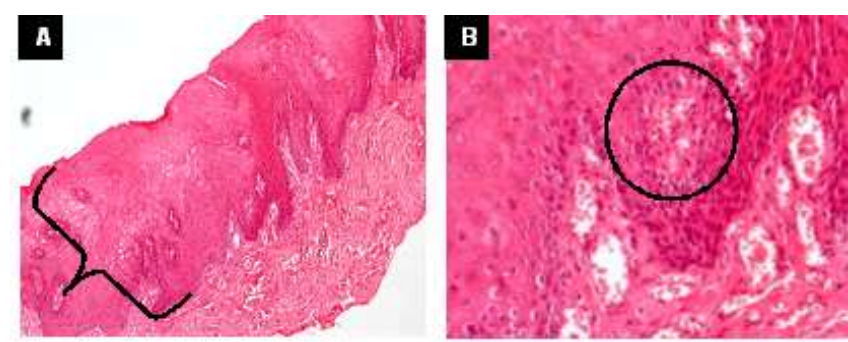

Figura 4: Exame histopatológico. A) Epitélio estratificado pavimentoso paraqueratinizado hiperplásico (chave). B) Intensa exocitose de leucócitos polimorfonucleares (círculo).

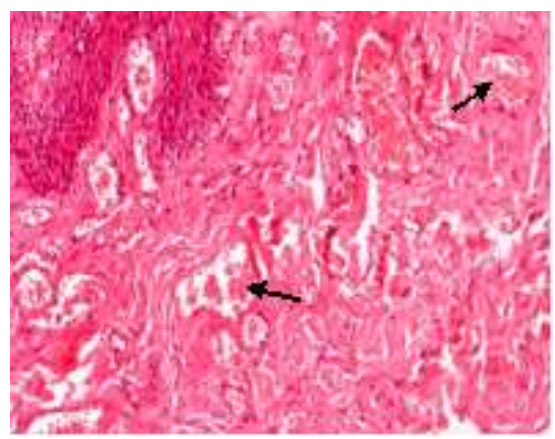

Figura 5: Tecido conjuntivo frouxo não modelado rico em capilares congestos (seta).

Uma semana após a cirurgia, a paciente retornou a clínica para a retirada dos pontos e a região mostrou-se totalmente cicatrizada (Figura 6 A). Após nove meses de proservação, não se verificou recidiva da lesão (Figura 6 B). $E$, decorridos 2 anos e 3 meses a região apresentava-se íntegra e sem sinal de recidiva (Figura 7).

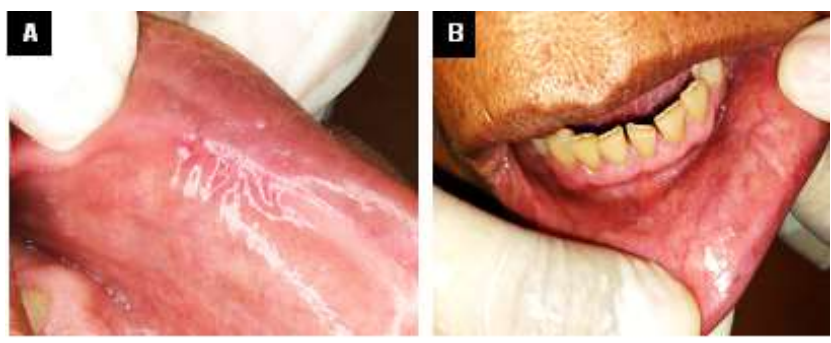

Figura 6: Aspecto da região labial após remoção cirúrgica. A) Sítio cirúrgico uma semana após a biopsia. B) Sítio cirúrgico nove meses após a biopsia.

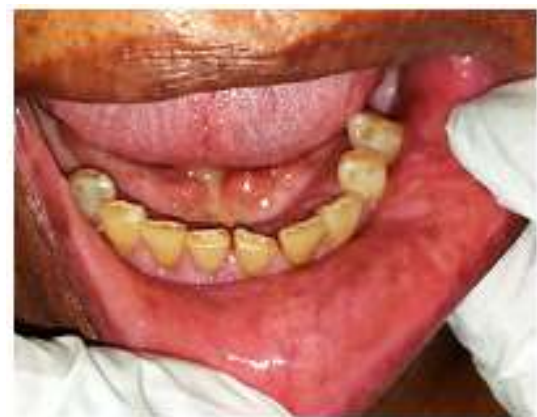

Figura 7: Sítio cirúrgico após 2 anos e 3 meses de proservação. DISCUSSÃO

O granulôma piogênico é uma neoplásica que pode surgir devido a influências hormonais, medicamentosas, traumas de baixa intensidade ou irritações crônicas na área acometida $^{1,24}$. A paciente em questão relatou que 0 dente posterior (primeiro pré-molar) traumatizava a região, neste caso, o hábito parafuncional de mordiscamento do lábio foi o fator etiológico da condição, o que corresponde com os estudos ${ }^{5,7}$.

O trauma crônico do lábio justifica o aparecimento da lesão na região, que devido a localização e história da doença atual poderia ser confundida com uma Mucocele, lesão causada por trauma mecânico em glândulas 
salivares menores, sendo o lábio inferior o local de maior ocorrência. O granuloma piogênico é um dos diagnósticos diferenciais da lesão ${ }^{25}$.

Clinicamente apresenta-se de forma exofítica, eritematosa, pediculada ou séssil, com superfície lisa ou lobulada e, geralmente ulcerada. Os aspectos clínicos da lesão observados mostram conformidade com a literatura ${ }^{5,10}$. No estudo epidemiológico de 191 casos de GP realizado por Avelar et al. ${ }^{1}$ (2008), no laboratório de Patologia Oral da Faculdade de Odontologia de Pernambuco, revelou que a maioria das lesões eram pediculadas correspondendo a $33,5 \%$ dos casos, corroborando com o trabalho de Rivero e Araújo $^{26}$ (1998), onde foram revisados 147 casos de GP, diagnosticados pelo Centro de Diagnóstico e Histopatologia (CDH) da Faculdade de Odontologia de Pelotas, dos quais em $74,7 \%$ a base era pediculada, porém a lesão da paciente em questão era séssil, confirmando caráter atípico. E apenas $21,5 \%$ dos pacientes relataram incômodo, assim como no caso da paciente, que relatou dor e desconforto.

$\mathrm{Na}$ anamnese, não soube informar $\mathrm{o}$ tempo exato de surgimento da lesão, apenas que havia aumentado de tamanho há cerca de dois meses. O processo evolutivo da lesão que variou de semanas a meses condiz com outros achados $^{11,26,27}$. Segundo Jafarzadeh et al. ${ }^{7}$ (2006), habitualmente o tamanho da lesão não ultrapassa $2,5 \mathrm{~cm}$ em sua maior dimensão, em concordância, a lesão obtinha $1 \mathrm{~cm}$.

Pesquisas comprovam que as faixas etárias mais acometidas são as de segunda e terceira décadas com um pico de prevalência em adolescentes e adultos jovens ${ }^{28,29}$. De acordo com Angelopoulous et al. ${ }^{30}$ (1971), um percentual de $60 \%$ das lesões ocorre em pessoas entre 11 e 40 anos. Entretanto, a paciente apresentou a lesão na sexta década de vida, mostrando que, apesar de ser uma década incomum, o GP pode acometer indivíduos de qualquer idade.

O local de maior ocorrência é a gengiva, representando $75 \%$ dos casos, contudo a lesão manifestou-se na região de lábio inferior $e$, apesar desta localização ser citada, possui uma baixa ocorrência, sendo um dos locais mais atípicos para o aparecimento da lesão ${ }^{1,29,31}$. A região anterior dos ossos gnáticos tem um acometimento de $(44,5 \%)$ e a região posterior $(33,4 \%)$ enquanto as demais localizações são acometidas em $(20,9 \%)$ dos $\operatorname{casos}^{2,30,32}$.

Os resultados da análise microscópica revelaram uma proliferação altamente vascular, sendo esta a principal característica da lesão. Constatou-se também um epitélio estratificado pavimentoso paraqueratinizado hiperplásico com intensa exocitose de leucócitos polimorfonucleares e um tecido conjuntivo frouxo não modelado, estando de acordo com os relatos da literatura ${ }^{5,18}$.

O tratamento consistiu na remoção cirúrgica da lesão, realizou-se uma biopsia excisional com pequena margem de segurança, com finalidade de evitar recidivas, sendo a lesão encaminhada para o laboratório de patologia bucal da UEA. As medidas realizadas seguiram propostas conforme alguns autores ${ }^{33,34,35}$. Segundo Giblin et al. ${ }^{36}$ (2007), a excisão completa é o tratamento mais recomendado, visto que tratamentos como cauterização, curetagem, excisão com laser cirúrgico, crioterapia ou fotocoagulação mostram ter baixa eficácia em eliminar a recorrência dessas lesões.

Uma reavaliação de 2 anos e 3 meses do pós-operatório não mostrou qualquer evidência de recidiva da lesão. Atribuísse também ao sucesso do caso a orientação e conscientização sobre o hábito parafuncional, em associação com o acompanhamento da região e cuidados realizados pela paciente.

CONCLUSÃO

Apesar da ocorrência incomum de granuloma piogênico no lábio inferior de um paciente senil, este relato ressalta o papel do cirurgião-dentista de ter conhecimento sobre essas lesões, pois uma interpretação errônea das características pode levar a um diagnóstico e tratamento incorretos. Durante o acompanhamento da paciente, não houve sinais de recidiva da lesão, mostrando que a escolha do tratamento (excisão cirúrgica) e orientação quanto ao hábito parafuncional, foram medidas eficazes para evitar sua recorrência.

AGRADECIMENTOS

Este trabalho foi apoiado pelo Serviço de Patologia Oral e Maxilofacial da Universidade do Estado do Amazonas (SEPAT - UEA), coordenado pelo Prof. Dr. Tiago Novaes Pinheiro, a quem agradecemos pela dedicação e colaboração para a conclusão deste.

\section{REFERÊNCIAS}

1. Avelar RL, Antunes AA, Carvalho RWS, Santos TS, Oliveira Neto PJ, Andrade ESS. Granuloma piogênico oral: um estudo epidemiológico de 191 casos. RGO. 2008;56(2):131-36.

2. Lawoyin JO, Arotiba JT, Dosumu OO. Oral pyogenic granuloma: a review of 38 cases from Ibadan, Nigeria. $\mathrm{Br} J$ Oral Maxillofac Surg. 1997;35(3):185-89.

3. Hullihen SP. Case of Aneurism by Anastomosis of the Superior Maxillare. The American journal of dental Science. 1844;4(3):160-62. 
4. Hartzell MB. Granuloma pyogenicum. J Cutan Dis Syph. 1904;22:520-25.

5. Neville BW, Damm DD, Allen CM, Bouquot JE. Patologia Oral e Maxilofacial. 3. ed. Rio de Janeiro: Elsevier; 2009. p. 509-66.

6. Gonçales ES, Damante JH, Fischer Rubira CM, Taveira LAA. Pyogenic granuloma on the upper lip: an unusual location. J Appl Oral Sci. 2010; 18(5):538-41.

7. Jafarzadeh H, Sanatkhani M, Mohtasham N. Gr anuloma Piogênico oral: uma revisão. J Oral Sci. 2006;48(4):167-75.

8. Kang HS, et al. Three Cases of Morsicatio Labiorum. Ann Dermatol. 2012;24(4):455-58.

9. Min KW, Park CK. Three Cases Report and a Review of the Literature. Korean J Pathol. 2009;43(2):174-76.

10. Gordón-Nuñez MA, Carvalho MV, Benevenuto TG, Lopes MFF, Silva LMM, Galvão HC. Oral Pyogenic Granuloma: A Retrospective Analysis of 293 Cases in a Brazilian Population. J Oral Maxillofac Surg. 2010;68(9):2185-88.

11. Vilmann $A$, Vilmann $P$, Vilmann $H$. Pyogenic granuloma: evaluation of oral conditions. $\mathrm{Br} \mathrm{J}$ Oral Maxillofac Surg. 1986;24(5):376-82.

12. Ramirez K, Bruce G, Carpinteiro W. Pyogenic granuloma: case report in a 9 year old girl. Gen Dent. 2002;50(3):280-81.

13. Karthikeya P, Mahima VG, Lahari K. Extragingi val pyogenic granuloma. Indian J Dent Res. 2006;17(4):199-202.

14. Kanda N, Watanabe S. Regulatory roles of sex hormones in cutaneous biology and immunology. J Dermatol Sci. 2005;38(1):1-7.

15. Silva-Sousa YT, Coelho CM, Brentegani LG, Vieira ML, Oliveira ML. Clinical and histological evaluation of granuloma gravidarum: case report. Braz Dent J. 2000;11(2):135-39.

16. Ojanotko-Harri AO, Harri MP, Hurttia HM, Sewoón LA. Altered tissue metabolism of progesterone in pregnancy gingivitis and granuloma. J Clin Periodontol. 1991;18(4): 262-66.

17. Brust AWA, Domingues JEG. Tratamento e proservação de nove meses em um paciente com granuloma piogênico: relato de caso. Rev Odontol UNESP. 2013;38(3):192-97.

18. Shafer WG, Hine MK, Levy BM. Diseases of the skin. In: Rajendran R. Shafer's Textbook of Oral Pathology. 5. ed. New Delhi: Reed Elsevier India Private Limited; 2006, p. 1103-107.

19. Bhaskar SN, Jacoway JR. Pyogenic granuloma - clinical features, incidence, histology, and result of treatment: report of 242 cases. J Oral Surg. 1966;24(5):391-98.

20. White JM, Chaudhry SI, Kudler JJ, Sekandari N, Schoelch ML, Silverman Jr. Nd: YAG and CO 2 laser therapy of oral mucosal lesions. J Clin Laser Med Surg. 1998;16(6):299-304.

21. Meffert JJ, Cagna DR, Meffert RM. Treatment of oral granulation tissue with the flashlamp pulsed dye laser. Dermatol Surg. 1998;24(8): 845-48.

22. Ishida CE, Ramos-e-Silva M. Cryosurgery in oral lesions. Int J Dermatol. 1998;37(4):283-85.

23. Moon SE, Hwang EJ, Cho KH. Treatment of pyogenic granuloma by sodium tetradecyl sulfate sclerotherapy. Arch Dermatol. 2005; 141(5):644-46.

24. Ramakrishnan $H$, Noorullah A, Venugopal L. A Clinical Report of Solitary Gingival Overgrowth in a Young Female Patient. J Pharm Bioallied Sci. 2019;11(2):491-94.

25. More CB, Bhavsar K, Varma S, Tailor M. Oral mucocele: a clinical and histopathological study. J Oral Maxillofac Pathol JOMFP. 2014;18(1):72.

26. Rivero ERC, Araújo LMA. Granuloma piogênico: uma análise clínico-histopatológica de 147 casos bucais. RFO-UPF. 1998;3(2): 55-61.

27. Tinoco PJ, Sanalzar N. Pyogenic granuloma vs. lobular capillary hemangioma. Histopathological analysis and epidemiology. Acta Odontol Venez. 1989;27(2-3):13-20.

28. Fortes TMV. Estudo epidemiológico de lesões proliferativas não neoplásicas da mucosa oral análise de 20 anos. Cienc Odontol Bras. 2002; 5(3):54-61.

29. Silveira EJD, Oliveira JMB, Galvão HC, Souza EL, Freitas RA. Granuloma piogênico. RGO. 2004;52(1):30-3.

30. Angelopoulos, AP. Pyogenic granuloma of the oral cavity: statistical analysis of its clinical features. J Oral Surg. 1971;29(12):840-47.

31. Sternberg SS, Antonioli DA, Carter D, Mills SE, Oberman $H$. Diagnostic Surgical Pathology. 3. ed. Filadélfia: Lippincot Williams e Wilkins; 1999. p. 169-74.

32. China ALP, Souza NMD, Amanajás TDA, Pedreira EN. Granuloma piogênico: relato de caso atípico em lábio inferior. Rev para med. 2010;24(3/4).

33. Carranza FA, Hogan EL. Periodontia clínica. 11. ed. Rio de Janeiro: Elsevier Saunders; 2012; p. 85-97.

34. Holmstrup P, Reibel J. Diagnóstico diferencial: tumores e cistos do periodonto. In: Lindhe J, Karring T, Lang NP. Tratado de periodontia clínica e implantologia oral. 4. ed. Rio de Janeiro: Guanabara Koogan; 2005. p. 290-308.

35. Shenoy SS, Dinkar AD. Pyogenic granuloma associated with bone loss in na eight year old child: a case report. J Indian Soc Pedod Prev Dent. 2006;24(4):201-3.

36. Giblin AV, Clover AJP, Athanassopoulos A, Budny PG. Pyogenic granuloma-the quest for optimum treatment: audit of treatment of 408 cases. J Plast Reconstr Aesthet Surg. 2007;60(9):1030-35. 


\section{CONFLITO DE INTERESSES}

Os autores declaram não haver conflitos de interesse

\section{AUTOR PARA CORRESPONDÊNCIA}

Gabrielly Melo Barbosa

Rua Catalunha, $n^{\circ} 26$, Bairro Nova Cidade

69097516 Manaus-AM, Brasil

E-mail: gmb.odo16@uea.edu.br

Submetido em 11/07/2021

Aceito em 01/09/2021 